\title{
Fluid management for the paediatric patient
}

Fluid replacement therapy in paediatric patients must be based on a knowledge of the normal physiology of infants and children and of the disturbances which may result from disease.

\section{The fluid compartments}

Large changes occur in total body water (TBW) from birth to adulthood and this has important clinical implications. The full-term neonate (TBW $80 \%$ of body weight) and older infants (TBW 65\% of body weight) have a greater proportion and also a different distribution of body water than do older children and adults (Figure 1). Much of the TBW of the infant is distributed to the extracellular fluid compartment (ECF). Due to this greater proportion of ECF $(40 \%)$, infants have a larger volume of distribution for drugs. Therefore, to achieve a given plasma concentration for water-soluble drugs (e.g., succinylcholine, nondepolarizing muscle relaxants, anticholinesterases, digoxin and most antibiotics) a higher loading dose on a per kilogram weight basis, is required. Adult values for TBW (50\%) and ECF (20\%) are reached during adolescence.

The size of the blood volume is inversely related to the age, see Table.

In addition to differences in TBW and its distribution, and the blood volume, premature and full-term infants have a much smaller proportion of body fat and muscle, but they have a larger percentage of vessel rich tissues.

\section{Normal fluid balance in infants and children}

Insensible water losses are higher in small infants. The higher level of alveolar ventilation results in increased water losses via the lungs, especially if inspired gases are low in humidity. The large body surface area to weight ratio plus the immature skin of the infant result in greater evaporative losses which are further increased by pyrexia or the use of warming lamps, phototherapy etc. Urine volumes are also higher in small infants; these are dictated by the limited ability of the kidney to concentrate urine (see below). These relatively higher rates of fluid turnover in infants and small children dictate that any interruption of fluid intake will more rapidly lead to depletion and dehydration.

\section{Renal function}

The healthy term infant is born with a similar number of nephrons to the adult, but they are small and functionally immature as evidenced by the limited renal function.

Renal blood flow is lower and the glomerular filtration rate (GFR) at birth is only $25-30 \%$ of the normal adult value. The GFR increases rapidly during the first few weeks of life, reaching $50 \%$ of adult value by $10-14$ days and $100 \%$ by the end of the first year. Infants born before 34 wk gestation demonstrate a less rapid increase in glomerular function due to delayed glomerular maturation.

The low GFR of early infancy limits the ability to excrete a water load, or to eliminate excess solute or drugs dependent upon renal clearance (pancuronium, dtubocurarine, aminoglycosides and many other antibiotics, $\mathrm{H}_{2}$-receptor blockers and digoxin). The lower clearance and longer half-life of these drugs may increase the potential for toxicity in the infant. Thus, although neonates may require a larger loading dose as a result of the increased volume of distribution, the dosing intervals may need to be increased to compensate for the decreased GFR.

Infants with hypoxia, hypothermia, congestive heart failure (CHF), respiratory distress syndrome (RDS) and hyperbilirubinaemia demonstrate a further decrease in GFR. Therefore meticulous attention to dosing intervals is necessary when caring for paediatric patients with these conditions and measurement of drug levels, where appropriate, may be very helpful. A peripheral nerve stimulator to assess neuro-muscular blockade is mandatory.

The normal serum creatinine concentration in the newborn is lower than that in adult patients $\left(0.4 \mathrm{mg} \cdot \mathrm{dl}^{-1}\right.$ vs $\left.1 \mathrm{mg} \cdot \mathrm{dl}^{-1}\right)$. This is a result of the high anabolic rate and small muscle mass/body weight ratio of the newborn.

Tubular function is less mature than glomerular function in the term neonate. The infant's ability to modify the glomerular filtrate in order to excrete or conserve substances (e.g., electrolytes, glucose, albumin, urea, bicarbonate and hydrogen ions) or to compensate for extremes of fluid balance, is impaired. This has some important clinical implications; continued excretion of sodium during a state of mild hyponatraemia, may lead to progressive neurological changes and seizures. Failure to con-

From the Department of Anesthesiology, Children's Hospital Los Angeles, Los Angeles, California. 


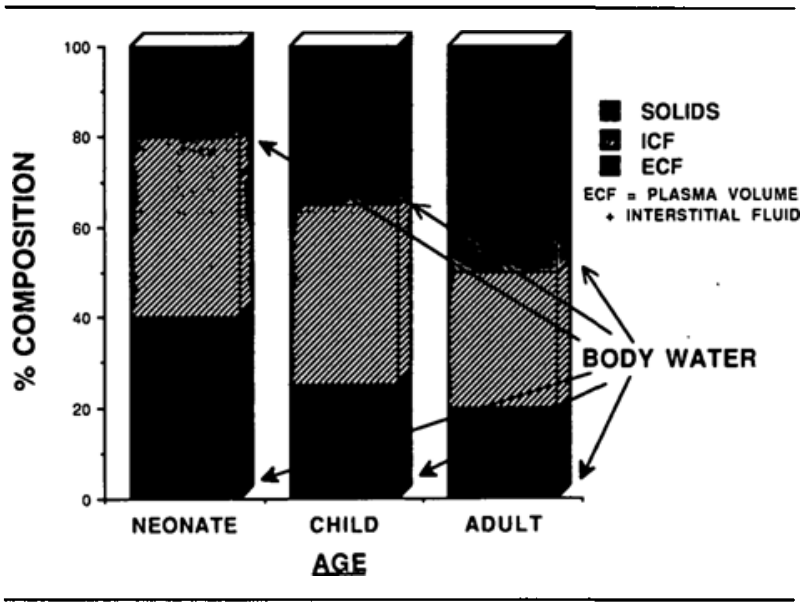

FIGURE Body Composition and Age

TABLE Blood volume and age

\begin{tabular}{ll}
\hline Preterm infant & $95 \mathrm{ml} \cdot \mathrm{kg}^{-1}$ body weight \\
Term neonate & $80 \mathrm{ml} \cdot \mathrm{kg}^{-1}$ \\
Infant & $75-80 \mathrm{ml} \cdot \mathrm{kg}^{-1}$ \\
Child & $65-75 \mathrm{ml} \cdot \mathrm{kg}^{-1}$ \\
\hline
\end{tabular}

serve sodium, especially in those infants who are dehydrated, may result in plasma hypo-osmolarity and sequelae (lethargy, muscle irritability, seizures, coma).

Glucosuria and proteinuria which may be seen frequently in infants, especially the preterm, are additional examples of tubular function immaturity. As a result of the limited tubular function, excess administration of glucose leads to glucosuria, the resultant osmotic diuresis potentially producing severe dehydration. Care must therefore be taken to administer only the appropriate required glucose-containing solutions to preterm infants. Proteinuria, in the form of albumin excretion, may occur in up to $20 \%$ of premature infants.

Newborn infants also have a lower renal threshold for bicarbonate than do adults, manifesting as a lower serum bicarbonate level $\left(20-21 \mathrm{mmol} \cdot \mathrm{L}^{-1}\right)$ and plasma $\mathrm{pH}$ of 7.35. The ability of the immature tubule to excrete acid is reduced in the preterm infant, thus impairing renal compensation of acidotic states.

The diluting and concentrating capabilities of the newborn's kidney mature rapidly during the early months of the first year, with renal tubular function reaching peak capacity by two to three years of age.

\section{Normal fluid and electrolyte requirements}

The higher fluid losses and greater metabolic activity of infants and small children dictate increased fluid requirements; maintenance fluid requirements of infants are as high as $6 \mathrm{ml} \cdot \mathrm{kg}^{-1} \cdot \mathrm{hr}^{-1}$, those of older children and adults approximately $1.5-2 \mathrm{ml} \cdot \mathrm{kg}^{-1} \cdot \mathrm{hr}^{-1}$. The electrolyte requirements of infants must accommodate the decreased ability of the kidney to conserve sodium. Infants will continue to lose sodium even when hyponatraemic. Daily potassium requirements are similar to those of sodium. Very small infants also cannot conserve bicarbonate.

\section{Perioperative fluid management}

Calculation of the volume and type of fluid required must take into consideration:

1 Dehydration present before preoperative fasting.

2 Fluid deficit incurred during preoperative fasting.

3 Maintenance fluid requirement during surgery.

4 Estimated extracellular fluid loss resultant upon surgical trauma.

5 Alterations in body temperature.

For short surgical procedures (under one hour) in otherwise healthy children, fluid is usually not needed $i v$ preoperatively if the preoperative deficit was small, fasting period short, and blood loss or tissue trauma is minimal, provided oral intake is likely to be re-established early in the postoperative period, e.g., hernia and hydrocoele repair, circumcision, minor plastic and orthopaedic procedures.

For surgical procedures of longer duration, when reestablishment of oral intake may be delayed:

1 An iv infusion is established.

2 Fluid is administered per- and postoperatively until oral intake is re-established.

$35 \%$ Dextrose in Ringer's lactate is usually given for simple procedures. This may cause hyperglycaemia during longer procedures and $2.5 \%$ dextrose in Ringer's lactate may be more appropriate.

4. For extensive surgery, especially in infants, it is advantageous to separate the administration of dextrose from other fluid therapy. Thus an infusion of 5 or $10 \%$ dextrose can be established at a rate which will deliver 4-6 $\mathrm{mg} \cdot \mathrm{kg}^{-1} \cdot \mathrm{min}^{-1}$. Blood glucose concentration should be checked periodically. Other fluids given to replace losses should be free of dextrose.

The hourly rate of infusion is based on daily maintenance requirements. Adjust the hourly rate if (a) factors affecting insensible fluid loss are present (e.g., increased body temperature) or (b) there are extrarenal losses (e.g., gastrointestinal).

Sufficient fluid should be given to compensate for preoperative fasting. The total volume to be administered during surgery is calculated by multiplying the number of hours (fasting + surgery) by the hourly maintenance requirement; for example, for a 10-kg child fasting for four hours and then undergoing an estimated four hour operative procedure, replacement and maintenance re- 
quirements would total $160+160=320 \mathrm{ml}$ (i.e., 8 $\left.\mathrm{ml} \cdot \mathrm{kg}^{-1} \cdot \mathrm{hr}^{-1}\right)$.

\begin{abstract}
Additional fluids
For surgical procedures causing considerable tissue trauma and/or blood loss, give additional fluids to replace extracellular fluid loss in blood or sequestered into damaged tissue. This deficiency should be replaced with a multiple-electrolyte solution, e.g., Ringer's lactate in which the electrolyte concentrations are similar to those in extracellular fluid. Abdominal surgery and surgery of the spine are associated with large fluid losses into the tissues and may require similarly large volumes of additional fluid $\left(6-10 \mathrm{ml} \cdot \mathrm{kg}^{-1}\right.$ or more). Thoracotomy is associated with much less translocation of fluid and hence lesser volumes will be required.

The adequacy of fluid replacement is best judged by continuous monitoring of the cardiovascular indices and urine output. If there is less than $1 \mathrm{ml} \cdot \mathrm{kg}^{-1} \cdot \mathrm{hr}^{-1}$ or urine the fluid infusion rate should be increased.
\end{abstract}

\section{Blood replacement}

A normal haemoglobin concentration is desirable in every case for elective surgery; below this, the risk of anaesthesia may be slightly increased and the decision whether to proceed must take into account all relevant factors in relation to the individual patient. In some patients elective surgery may be delayed until the anaemia has been investigated and treated. In some anaemic patients, surgery may be more urgent and anaesthesia for these patients must be with a technique that is compatible with their anaemia. When surgery cannot be delayed despite a very low $\mathrm{Hb}$ value, packed cells should be infused preoperatively. Approximately $4 \mathrm{ml}$ packed cells $\cdot \mathrm{kg}^{-1}$ is required to raise the $\mathrm{Hb}$ concentration by $1 \mathrm{~g} \cdot \mathrm{dl}^{-1}$.

$\mathrm{Hb}$ content of:

Stored whole blood $=12 \mathrm{~g} \cdot \mathrm{dl}^{-1}$

Packed cells $=24 \mathrm{~g} \cdot \mathrm{dl}^{-1}$

Buffy-coat-poor washed cells $=28 \mathrm{~g} \cdot \mathrm{dl}^{-1}$

When blood loss of $10 \%$ of the estimated blood volume (EBV) or greater might be expected, the patient's blood should be grouped and cross-matched for a volume depending on expected losses. Insert à CVP line preoperatively in patients who are hypovolaemic and/or may require massive blood replacement during surgery.

At commencement of the operation, record on the anaesthesia chart the EBV and the preoperative $\mathrm{Hb}$ level.

\section{Assessment of blood loss}

Accurate estimates of blood loss must be maintained throughout the operation:
Monitor CVS indices; in infants, the systolic BP is the most reliable indicator of blood volume.

Measure blood loss from the surgical site:

(a) All sponges should be weighed before they dry out. This is simple and accurate (assume $1 \mathrm{~g}=1 \mathrm{ml}$ blood and subtract the known dry weight).

(b) Measure blood from suction (in graduated flasks).

(c) Estimate blood on drapes.

Chart the running total continually.

$\mathrm{Be}$ aware of the possibility that blood losses may accumulate in body cavities (e.g., peritoneum, pleura).

\section{Blood transfusion}

The decision whether to transfuse blood must be based on the preoperative $\mathrm{Hb}$ concentration, the measured surgical blood loss, and the patient's cardiovascular response. The need for blood transfusion can be determined more accurately from serial Hct measurements (normally the Hct should be maintained at or above $30 \%$ in infants).

Each unit of blood must be checked against the patient's identity bracelet and mixed well by repeated inversion of the bag. Blood should be warmed to $37^{\circ} \mathrm{C}$ before administration but it should not be heated above $38^{\circ} \mathrm{C}$ or it may be damaged.

Calcium gluconate is sometimes necessary during massive transfusion in children and may be given $(0.1 \mathrm{ml}$ of a $10 \%$ solution $/ \mathrm{kg}$ ) if persistent hypotension follows apparently adequate volume replacement in infants. Hyperkalaemia may become dangerous during the very rapid infusion of large volumes of blood to children.

If it becomes apparent that massive blood transfusion will be required (i.e., $>75 \%$ of EBV), coagulation incides should be monitored. Platelet counts, prothrombin time, and partial thromboplastin time together with tests for fibrinolysis (determination of fibrin split products) should be repeated at least after every $50 \%$ blood volume replacement. It is helpful to have a preoperative platelet count if massive transfusion is a possibility. A low initial count will indicate the need for early platelet transfusion. Platelet counts of $\left\langle 65,000 \cdot \mathrm{mm}^{-3}\right.$. will increase clinical bleeding and should be corrected. In practice, if platelets are being monitored during massive replacement, this means that platelets should be ordered as the count goes below 100,000. Infusion of one unit of platelet concentrate per $5 \mathrm{~kg}$ body weight will increase the platelet count by $30-40,000$ per $\mathrm{cmm}$. Platelets must be stored at room temperature and not refrigerated. Other documented deficiencies that become apparent should be dealt with by appropriate therapy (e.g., fresh frozen plasma or appropriate blood component therapy). 


\section{References}

1 Berry FA. Practical aspects of fluid and electrolyte therapy. In: Berry FA (Ed.). Anesthetic Management of Difficult and Routine Pediatric Patients. 2nd ed. New York: Churchill Livingstone, 1990, 89-120.

2 Bourke DL, Smith TC. Estimating allowable hemodilution. Anesthesiology 1974; 41: 609-12.

3 Cote CJ, Liu LM, Szyfelbein SK, Goudzouzian NG, Daniels $A L$. Changes in serial platelet counts following massive blood transfusions in pediatric patients. Anesthesiology 1985; 62: 197-201.

4 Consensus conference: Perioperative red blood cell transfusion. JAMA 1988; 260: 2700-3.

5 Filston $H C$, Johnson DG. Percutaneous venous cannulation in neonates and infants: a method for catheter insertion without "cut-down." Pediatrics 1971; 48: 896-901.

6 Guignard $J P$. Renal function in the newborn infant. Pediatr Clin North Am 1982; 29: 777-90.

7 Hall SC, Przybylo HJ, Roth AJ. 5\% and 10\% dextrose intravenous solutions during neonatal surgery. Anesthesiology 1988; 69: A738.

8 Leake RD. Perinatal nephrobiology: a developmental perspective. Clin Perinatol 1977; 4: 321-49.

9 Welborn LG, Hannallah RS, McGill WA, Ruttiman UE, Hicks JM. Glucose concentrations for routine intravenous infusion in pediatric outpatient surgery. Anesthesiology 1987; 67: 427-30. 


\section{L'apport hydrique en anesthésie pédiatrique}

En anesthésie pédiatrique, le remplacement liquidien repose sur la connaissance de la physiologie néo-natale et infantile et des dérangements provoqués par la maladie.

\section{Les compartiments hydriques}

Des mouvements importants de l'eau totale de l'organisme $\left(\mathrm{H}_{2} \mathrm{O}\right.$ tot) surviennent entre la naissance et l'âge adulte. Le nouveau-né à terme $\left(\mathrm{H}_{2} \mathrm{O}\right.$ tot $=80 \%$ du poids) et le nourrisson plus âgé $\left(\mathrm{H}_{2} \mathrm{O}\right.$ tot $=65 \%$ du poids) dont la proportion d'eau est plus importante, ont une distribution différente de celle de l'enfant plus âgé et de l'adulte (Figure). Une bonne partie de l' $\mathrm{H}_{2} \mathrm{O}$ tot du nourrisson appartient au compartiment extracullaire (liquide extracellulaire: LEC). A cause de cette proportion plus élevée de LEC (40\%), les nourrissons possèdent un plus grand volume de distribution pour les drogues. Par conséquent, pour atteindre une concentration donnée, les drogues hydrosolubles (ex. la succinylcholine, les myorelaxants non dépolarisants, les anti-cholinestérasiques, la digoxine et la plupart des antibiotiques) requièrent une dose de charge plus forte par kilogramme de poids. Les valeurs adultes d' $\mathrm{H}_{2} \mathrm{O}$ tot et de LEC (20\%) sont atteintes à l'adolescence.

Le volume sanguin est inversement proportionnel à l'âge (voir Tableau).

En plus des différences de pourcentage d'eau totale, de distribution hydrique et de volume sanguin, les prématurés et les nouveaux-nés à terme ont une plus faible proportion de graisse et de muscle, mais un pourcentage plus élevé de tissus riches en vaisseaux.

\section{L'équilibre hydrique du nourrisson et de l'enfant}

Le pertes insensibles sont plus importantes chez le jeune enfant. A cause du niveau de ventilation alvéolaire plus élevé de l'enfant, la perte d'eau pulmonaire augmente surtout lorsque les gaz inspirés sont secs. Chez le nourrisson, avec l'immaturité cutanée, le rapport surface/poids plus élevé entraîne des pertes plus importantes par évaporation, lesquelles sont amplifiées par les lampes chauffantes, la photothérapie, etc. Les volumes urinaires sont aussi plus importants chez le nourrisson à cause de son incapacité à concentrer l'urine (voir plus bas). Chez le nouveau-né et le nourrisson, la vitesse accélérée des mutations liquidiennes provoque après l'arrêt de l'hydratation une déplétion et une déshydratation rapides.

\section{La fonction rénale}

Le nouveau-né en bonne santé et à terme naît avec le même nombre de néphrons que l'adulte. Les néphrons sont petits et fonctionnellement immatures comme en témoigne une fonction rénale limitée.

A la naissance, le débit sanguin rénal est plus bas et le taux de filtration glomérulaire (TFG) ne représente que 25 à 30\% des valeurs adultes normales. Le TFG augmente rapidement pendant les premières semaines de vie et atteint $50 \%$ de la valeur adulte entre le $10 \mathrm{e}$ et $14 \mathrm{e}$ jour et $100 \%$ à la fin de la première année. Chez les enfants nés avant 34 sẻmaines de gestation, la fonction glomérulaire progresse plus lentement par retard de maturation gloménulaire.

La baisse initiale du TFG limite la capacité d'excrétion de la charge hydrique et d'élimination des solutés en excès et des drogues dont l'élimination dépend de la clairance rénale (le pancuronium, la d-tubocurarine, les aminoglycosides et plusieurs autres antibiotiques, les inhibiteurs $\mathrm{H}_{2}$ et la digoxine). Une clairance plus basse et une demivie prolongée augmentent la toxicité potentielle. Ainsi, bien que les nouveaux-né puissent avoir besoin d'une dose de charge plus forte à cause du volume accru de distribution, il faut augmenter les intervalles entre les doses pour compenser pour la baisse du TFG.

Chez les enfants hypoxiques, hypothermiques, insuffisants cardiaques ou en détresse respiratoire et hyperbilirubinémiques, le TFG baisse encore plus. Chez ces enfants, il faut titrer méticuleusement les intervalles d'administration. La mesure des concentrations plasmatiques peut être utile. On devrait toujours utiliser un stimulateur nerveux périphérique pour évaluer le bloc neuromusculaire.

La créatinémie normale du nouveau-né est plus basse que celle de l'adulte $\left(0,4 \mathrm{mg} \cdot \mathrm{dl}^{-1}\right.$ vs $\left.1 \mathrm{mg} \cdot \mathrm{dl}^{-1}\right)$ et résulte du catabolisme élevé et d'un bas rapport muscle/graisse.

Chez le nouveau-né à terme, la fonction tubulaire est moins mature que la fonction glomérulaire. Chez le nourrisson, on peut mettre en évidence une altération de la capacité de modifier le filtrat glomérulaire pour l'excrétion ou la rétention de certaines substances (ex. les électrolytes, le glucose, l'albumine, l'urée, le bicarbonate et l'ion hydrogène); il compense aussi difficilement pour les extrèmes de l'equilibre hydrique. Les implications cliniques de ces caractéristiques sont importantes: l'excrétion continue de sodium pendant les états d'hyponatrémie légère peut provoquer une atteinte neurologique progres- 


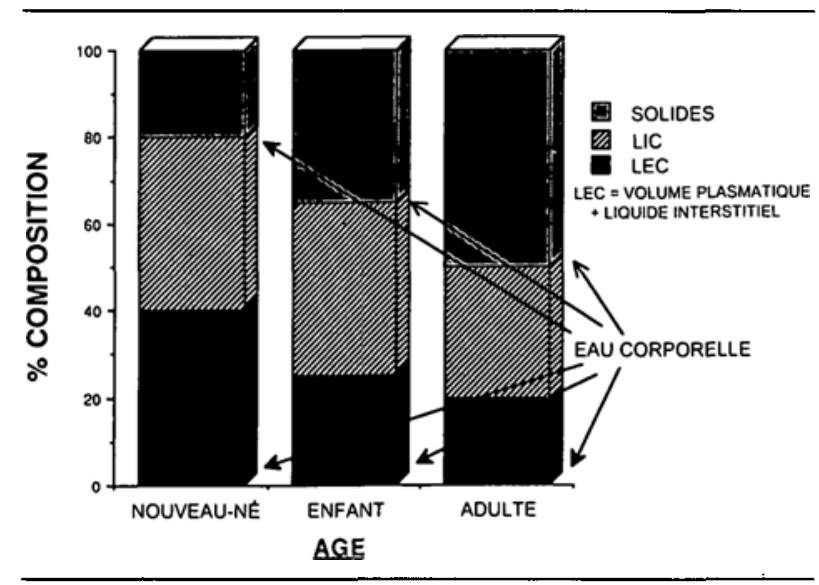

FIGURE Composition de l'organisme et âge

TABLEAU Volume sanguin et âge

\begin{tabular}{ll}
\hline Prématuré & $95 \mathrm{ml} \cdot \mathrm{kg}^{-1}$ \\
Nouveau-né à terme & $80 \mathrm{ml} \cdot \mathrm{kg}^{-1}$ \\
Nourisson & $75-80 \mathrm{ml} \cdot \mathrm{kg}^{-1}$ \\
Enfant & $65-75 \mathrm{ml} \cdot \mathrm{kg}^{-1}$ \\
\hline
\end{tabular}

sive et des convulsions. Lincapacité de conserver le sodium, spécialement chez l'enfant déshydraté, peut conduire à l'hypo-osmolarité plasmatique et à ses manifestations, dont la léthargie, l'irritabilité musculaire, les convulsions et le coma.

La glucosurie et la protéinurie, fréquentes chez le nourrisson surtout le prématuré, sont encore des manifestations de limmaturité de la fonction tubulaire. Comme résultat de la limitation de la fonction tubulaire, l'administration excessive de glucose mène à la glucosurie, avec la conséquence possible d'une diurèse osmotique avec déhydratation grave. Il faut donc prendre soin de n'administrer aux prématurés que des solutés glucosés appropriés. La protéinurie peut survenir par excrétion d'albumine chez $20 \%$ des prématurés.

Le nouveaux-né possède aussi pour les bicarbonates un seuil rénal inférieur à celui de l'adulte, comme le montre son taux de bicarbonate plasmatique (20-21 $\mathrm{mmol} \cdot \mathrm{L}^{-1}$ ) et son $\mathrm{pH}$ de 7,35 . La capacité tubulaire d'excrétion des acides du prématuré est réduite, ce qui l'empêche de compenser efficacement pour l'acidose.

Les capacités de dilution et de concentration rénales du nouveau-né atteignent une maturité rapide pendant les premiers mois et la fonction tubulaire atteint son niveau d'activité le plus élevé entre deux à trois ans.

\section{Besoins hydroélectrolytiques}

Chez le nourrisson et le jeune enfant, des pertes de liquides et l'activité métabolique plus importante augmentent les besoins hydriques: le maintien de l'hydratation du nourrisson nécessite des volumes aussi élevés que 6 $\mathrm{ml} \cdot \mathrm{kg} \cdot \mathrm{h}^{-1}$, alors que ceux des enfants plus âges et des adultes varient de 1,5 à $2 \mathrm{ml} \cdot \mathrm{kg} \cdot \mathrm{h}^{-1}$. Les besoins électrolytiques doivent s'ajuster à la diminution de la capacité du rein à conserver le sodium. Les enfants continuent à perdre du sodium même sils sont hyponatrémiques. Les besoins en potassium sont identiques à ceux du sodium. Les prématurés ne conservent pas le bicarbonate.

\section{Les apports hydroélectrolytiques}

Pour le calcul du volume et le choix du type de soluté, il faut tenir compte de:

1 la déshydratation présente avant l'intervention;

2 du déficit hydrique lié au jeûne préopératoire;

3 des besoins liquidiens d'entretien pendant la chirurgie;

4 de la perte de liquide extracellulaire résultant du traumatisme chirurgical;

5 des changements de température corporelle.

Pour les interventions de courte durée (moins d'une heure) chez des enfant non tarés, des liquides $i v$ ne sont pas nécessaires pendant l'opération si le déficit préopératoire est minime, en autant qu'on pourra rapidement rétablir l'alimentation orale à la période postopératoire, ex. pour une cure d'hydrocèle ou de hernie, une circoncision, une chirurgie plastique ou orthopédique mineure.

Pour les interventions de plus longue durée, lorsque l'alimentation orale sera retardée:

1 Installer une perfusion

2 Administrer des solutés pendant et après l'opération jusqu'à la reprise de l'alimentation orale.

3 Administrer du dextrose 5\% dans du lactate de Ringer pendant les interventions simples. Cette formule peut provoquer de l'hyperglycémie pendant les interventions prolongées; du dextrose $2.5 \%$ dans le lactate est alors préférable.

4 Pour la chirurgie lourde, il est avantageux chez l'enfant de séparer le dextrose des autres solutés. Ainsi on peut établir une perfusion de dextrose 5 ou $10 \%$ à la vitesse de $4-6 \mathrm{ml} \cdot \mathrm{kg} \cdot \mathrm{min}^{-1}$. Il faut vérifier la glycémie périodiquement. Les autres liquides administrés pour remplacer les pertes ne devraient pas contenir de dextrose.

La vitesse d'administration horaire est basée sur les besoins quotidiens. Ajuster la vitesse horaire si a) des facteurs qui affectent les pertes insensibles sont présents (ex. l'hyperthermie) ou b) on rencontre des pertes autres que rénales (ex. gastrointestinales).

Il faut administrer des liquides en quantité suffisante pour compenser pour le jeûne préopératoire. Le volume total à administrer pendant la chirurgie est calculé en multipliant le nombre d'heures (jeûne + chirurgie) par les besoins horaires d'entretien; par exemple, pour un enfant de dix kilos à jeun depuis quatre heures et subissant une intervention de quatre heures, les volumes de remplacement et d'entretien seront de $160+160=320 \mathrm{ml}$ (c.-à-d.) $8 \mathrm{ml} \cdot \mathrm{kg} \cdot \mathrm{h}^{-1}$. 


\section{Liquides supplémentaires}

Pour les interventions traumatiques ou sanglantes, il faut administrer un supplément de liquide pour compenser la perte sanguine et les liquides séquestrés dans les tissus lésés (troisième secteur). Ce type de déficit est remplacé par une solution poly-électrolytique, ex. une solution de lactate de Ringer dont la concentration électrolytique est la même que celle du liquide extracellulaire. La chirurgie abdominale et vertébrale s'accompagnent d'extravasations importantes de liquide vers les tissus et peut nécessiter de grands volumes supplémentaires $(6-10 \mathrm{ml} \cdot \mathrm{kg}$ ou plus). La thoracotomie s'accompagne de moins de mutations liquidiennes et requiert moins de remplacement.

L'efficacité du remplissage vasculaire est évaluée par le monitorage continu des signes cardiovasculaires et $\mathrm{du}$ débit urinaire. Si la diurèse est moindre que 1 $\mathrm{ml} \cdot \mathrm{kg} \cdot \mathrm{h}^{-1}$, il faut augmenter la perfusion de liquide.

\section{Remplacement du sang}

Dans tous les cas de chirurgie réglée, une concentration normale en hémoglobine $(\mathrm{Hb})$ est préférable: une concentration inférieure à la normale, augmente le risque chirurgical légèrement et la décision d'effectuer l'intervention tiendra compte de tous les facteurs appropriés à chaque cas individuel. Chez certains, la chirurgie réglée peut être retardée pour déterminer l'étiologie de l'anémie et la traiter. Chez d'autres, la chirurgie peut être plus urgente et il faudra alors administrer une technique anesthésique appropriée à l'anémie. Quand la chirurgie ne peut être retardée malgré une $\mathrm{Hb}$ basse, il faut transfuser des concentrés érythrocytaire à la période préopératoire. Environ $4 \mathrm{ml} \cdot \mathrm{kg}^{-1}$ de concentré érythrocytaire sont requis pour élever le taux d'Hb de $1 \mathrm{~g} \cdot \mathrm{dl}^{-1}$ :

Contenu en $\mathrm{Hb}$ :

du sang conservé complet $=12 \mathrm{~g} \cdot \mathrm{dl}^{-1}$

du concentré érythrocytaire $=24 \mathrm{~g} \cdot \mathrm{dl}^{-1}$

des érythrocytes lavés $=28 \mathrm{~g} \cdot \mathrm{dl}^{-1}$

Quand on prévoit une perte sanguine de $10 \%$ du volume sanguin circulant ou plus, il faut préparer une quantité de sang compatible équivalente à la perte sanguine anticipée. Insérer une TVC à la période préopératoire aux patients hypovolémiques et à ceux qui auront besoin de transfusions massives pendant la chirurgie.

$\mathrm{Au}$ début de l'opération, inscrire la valeur du volume sanguin circulant au dossier ainsi que le taux d'hémoglobine préopératoire.

\section{Evaluation des pertes sanguines}

Il faut suivre l'évolution des pertes sanguines pendant toute la durée de l'intervention:
Monitorer les indices cardiovasculaires chez l'enfant, la TA systolique demeure le meilleur indice du volume sanguin.

a) Il faut peser toutes les éponges avant qu'elles ne sèchent. Cette méthode est simple et précise (assumer $1 \mathrm{~g}=1 \mathrm{ml}$ de sang et soustraire le poids sec connu).

b) Mesurer le sang aspiré des bouteilles graduées.

c) Evaluer la quantité du sang contenu dans les champs. Maintenir ce décompte en tout temps.

Prendre en compte le sang accumulé dans les cavités (ex. péritoine, plèvres).

\section{Les transfusions}

La décision de transfuser doit reposer sur le taux d'Hb préopératoire, la quantité du sang perdu et la réaction cardiovasculaire. Le besoin de transfusion s'evalue avec plus de précison si on fait des mesure répétées de l'hématocrite (normalement chez l'enfant, on maintien l'Hct $\geq 30 \%$ ).

Verifier l'identité de chacun des sacs de sang avec le bracelet d'identification de l'enfant. Le sang doit être réchauffé à $37^{\circ} \mathrm{C}$ mais jamais à $38^{\circ} \mathrm{C}$, ce qui pourrait l'endommager.

Lors des transfusions massives, il est quelquefois nécessaire d'administrer du gluconate de calcium $\left(0,1 \mathrm{ml} \cdot \mathrm{kg}^{-1}\right.$ de la solution à 10\%) si l'hypotension persiste malgré un remplissage adéquat. La transfusion sanguine massive et rapide peut causer une hyperkaliémie sérieuse.

Sil devient apparent que des transfusions massives seront requises (c.-à-d. $>30 \%$ du volume sanguin circulant), il faut monitorer l'hémostase. La numération des plaquettes, le temps de prothrombine et de thromboplastine partielle avec les épreuves de fibrinolyse (produits de dégradation de la fibrine) doivent être répétés après chaque remplacement du volume sanguin de $50 \%$. Si on craint devoir transfuser massivement, il est utile de faire une numération plaquettaire en préopératoire. Une numération initiale basse nécessite des transfusions précoces de plaquettes. Avec des plaquettes au nombre 65,000 ou moins, le saignement visible pourra augmenter. En pratique, si on monitorise les plaquettes pendant un remplacement massif, il faut commander des plaquettes de donneur dès que la numération chute sous les 100,000 . $\mathrm{La}$ transfusion d'une unité de concentré plaquettaire par $5 \mathrm{~kg}$ de poids augmentera le nombre de 30-40,000 par $\mathrm{ml}$. Les plaquettes doivent être conservées à la température de la pièce. Tout autre déficience prouvée devrait être traitée spécifiquement (ex. plasma frais congelé ou la fraction de sang appropriée).

\section{Références}

(Voir page R90) 